This item was submitted to Loughborough's Research Repository by the author.

Items in Figshare are protected by copyright, with all rights reserved, unless otherwise indicated.

\title{
Computer vision for textured yarn interlace (Nip) measurements at high speeds
}

\section{PLEASE CITE THE PUBLISHED VERSION}

http://dx.doi.org/10.1016/S0957-4158(00)00056-8

\section{PUBLISHER}

(C) Elsevier Science Ltd

\section{VERSION}

AM (Accepted Manuscript)

\section{PUBLISHER STATEMENT}

This work is made available according to the conditions of the Creative Commons Attribution-NonCommercialNoDerivatives 4.0 International (CC BY-NC-ND 4.0) licence. Full details of this licence are available at: https://creativecommons.org/licenses/by-nc-nd/4.0/

\section{LICENCE}

CC BY-NC-ND 4.0

\section{REPOSITORY RECORD}

Millman, Michael P., Memis Acar, and Michael R. Jackson. 2019. "Computer Vision for Textured Yarn Interlace (nip) Measurements at High Speeds”. figshare. https://hdl.handle.net/2134/22046. 


\title{
Computer Vision for Textured Yarn Interlace (Nip) Measurements at High Speeds.
}

\author{
M.P. Millman, M. Acar \& M.R. Jackson \\ Department of Mechanical Engineering \\ Loughborough University \\ Leicestershire, LE11 3TU, UK
}

\begin{abstract}
This paper deals specifically with interlace measurements of intermingled, false-twisttextured yarns. A system has been built which is capable of analysing yarns at slow speed / high resolution, and also at high speeds / low resolution. An outline description of the system is given here, along with descriptions of techniques used in the hardware and software which substantially improved the system performace. To monitor interlace, a recently developed signal processing algorithm is explained which enables reliable interlace detection, and is effective against noise. Several tests are carried out, which prove the ability of the system to match the results from careful manual inspection of the yarn. The final test is to run the yarn at a high speed, and to compare the results with the slow speed analysis. The results show that at high speeds, the system is reliable. A discussion of some hardware requirements for an on-line optimised commercial system is given.
\end{abstract}

\section{INTRODUCTION}

Computer vision is becoming more affordable as both a research and in-process quality control tool. It has the advantage of providing increased amounts of characterisation of the test product, at high speeds and resolutions, as well as being non-contact.

Synthetic continuous filament yarns are usually textured prior to their successive usage in further processes, such as weaving and knitting [1]. The purpose of conventional texturing is to impart texture to the regularly packed, flat filament yarn to give comfort and aesthetics to the finished product. The most common texturing technique is the false-twist texturing process which produces 'stretch' yarns that are highly extensible even under very low loads. This arises from heat setting the twisted deformations introduced to the yarn during the texturing process.

False-twist textured yarns lack inter-filament cohesion, and consequently a number of difficulties are observed during unwinding and fabric forming processes. One of the modern techniques of imparting such cohesion is air-intermingling (interlacing) of false-twist textured yarns. Intermingling is a process which deploys a nozzle to create a very turbulent high speed air flow. This imparts regular but intermittently entangled nodes to the open structure of the textured yarn which are commonly known in industry as 'nips' [2]. Figure 1 shows an SEM image of a typical intermingled yarn showing entangled sections (nips) and open sections between them. 


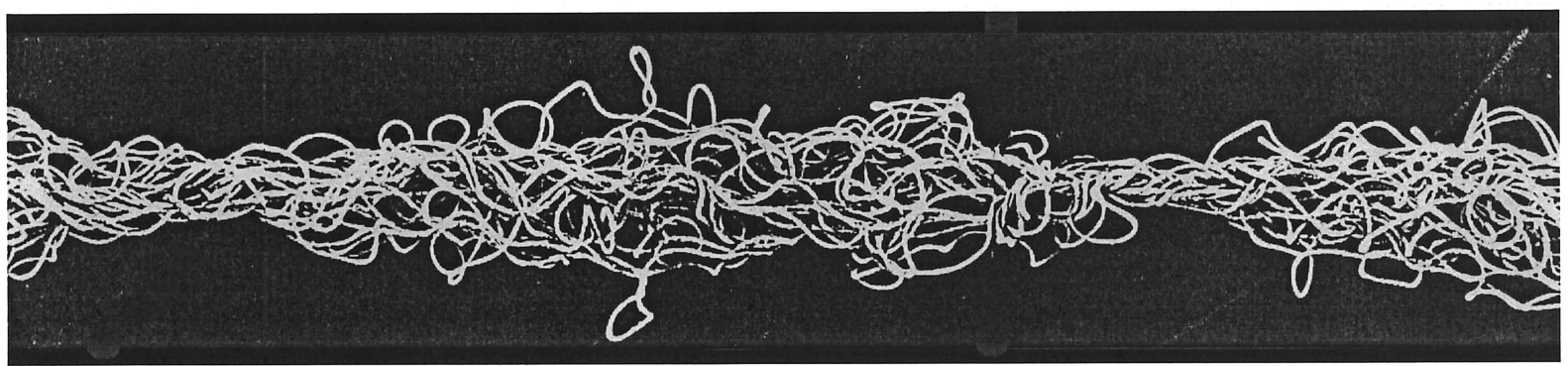

Figure 1. SEM image of a false-twist textured, intermingled yarn

The frequency and regularity of the nips together with their stability under applied loads are important criteria in the assessment of the performance of mingling nozzles and the quality of the intermingled yarns.

\section{SYSTEM OUTLINE}

The overall system is divided into two main parts; the yarn transport and the yarn imaging/analysis. For each part, a separate PC computer is used, and these are connected together. The imaging PC controls all operations, and sends instructions to the transport control PC, which in turn can operate in a stand alone fashion if necessary.

\subsection{Yarn imaging}

The yarn is imaged using a 1024 pixel line scan CCD camera which scans up to $10 \mathrm{kHz}$. For a yarn travelling at $10 \mathrm{~m} / \mathrm{s}$ the scan to scan resolution is $1 \mathrm{~mm}$. Two orthogonal views of the yarn in the same cross sectional plane are used to characterise the yarn. A mirror inclined at $45^{\circ}$ to the viewing plane achieves this as shown in Figure 2. This technique has been shown to provide more accurate evenness data for staple fibre yarns [3]. The magnification is set to 1 , giving a pixel resolution of 13 microns.

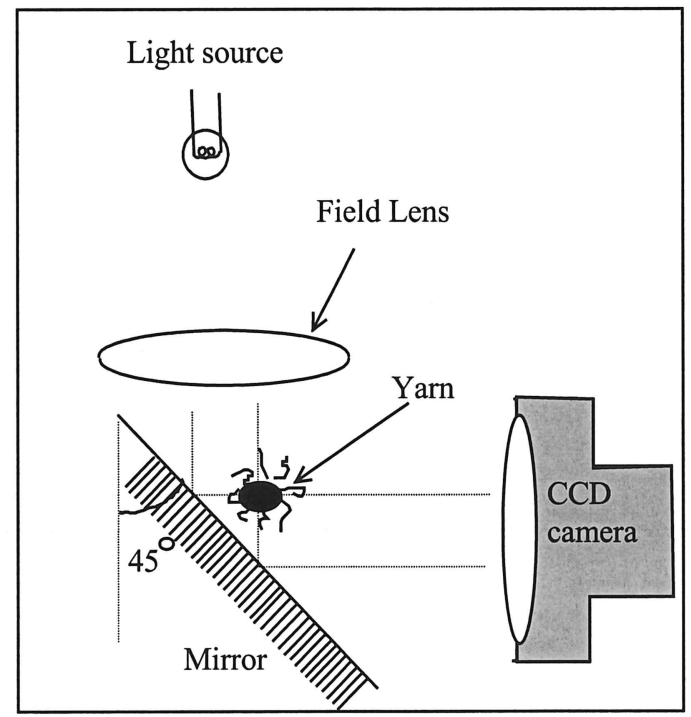

Figure 2: Arrangement for obtaining two orthogonal views of yarn image 
A key optical technique when imaging yarns for computer vision is to use a field lens before the yarn. This effectively focuses the light source ( 30 watt halogen bulb) onto the aperture of the camera, which causes a much larger degree of light intensity to be seen at the CCD. Furthermore, the light intensity profile across the CCD is much more constant than without a field lens and this is critical when attempting to image loose fibres and broken filaments (Figure $3 \mathrm{a} \& \mathrm{~b}$ ). The alternative to this is to use a diffuser instead of the field lens, but this absorbs a significant proportion of light, and does not give as even an illumination.

light intensity on CCD

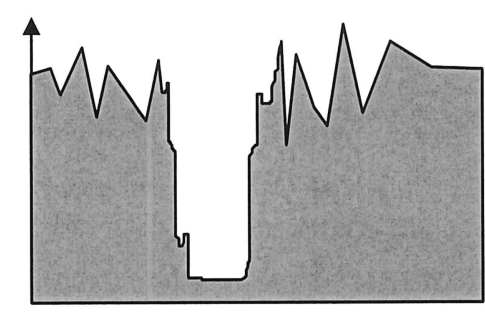

(a)

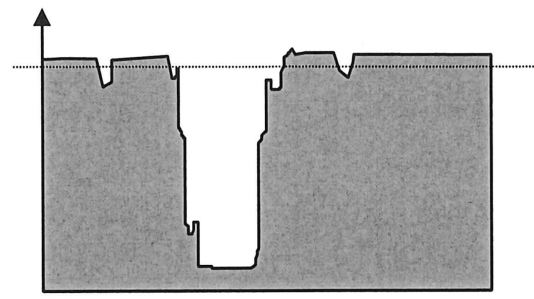

(b)

Figure 3 - Intensity profile of a yarn image without using a field lens or diffuser, the dotted line in (b) represents a threshold used for detecting broken filaments/loose fibres.

The camera is interfaced to a PC using an Optimum Vision OVB10 board. This binarises the incoming grey level analogue signal, and sends the edge data to the PC on a FIFO stack system. For nip detection, the grey level data is not required, and binarising the image is all that is required from an imaging point of view. This isn't the case for yarn hairiness or evenness analysis [5]

An important part of the software is the acquisition of the edge data from the OVB10, and is written in $\mathrm{C}$ code. However, the part which reads data from the OVB10 board had to be optimised to the full, in order to be quick enough to receive the data. The original $\mathrm{C}$ code, assembler output was taken, and modified by using most of the Intel 80386 register set, with careful thought given to instruction timings etc. The result is faster code, and the image acquisition is less prone to saturation if there is too much edge data from the OVB10. This technique can substantially improve the performance of many software based real time applications, where the code is written in a high/medium level language.

\subsection{Slow Speed Yarn Transport}

An off-line computer controlled yarn transport has been developed which can transport the yarn at slow speeds $(7 \mathrm{~cm} / \mathrm{s})$, but with highly responsive start / stop properties using stepper motors. The tension of intermingled yarns affects the nip dimensions as the yarns stretch. Therefore a closed loop PID tension control was built, such that the tension remained constant, and could be held steady at low tensions $(\sim 5 \mathrm{cN})$. The tension data is also monitored, and sent to the imaging PC at the end of a run to check if there were no glitches in the yarn transport. 
The yarn is engaged to the stepper motors by means of solenoid actuated capstan rollers, which pinch the yarn against the motor shaft. The two motors are at either end of the imaging area. The motor situated after the imaging area is run at constant speed, whereas the motor before the area is controlled by the tension controller software (Figure 4).

\subsection{High speed yarn transport}

A high speed yarn transport (up to $10 \mathrm{~m} / \mathrm{s}$ ) was made such that yarns could be tested at high production speeds. The results could be compared to those at lower speeds to show the effect of reducing the scan to scan resolution .

Two printed circuit DC servo motors were used in the same fashion as the stepper motors above i.e. with one after and one before the imaging area. The motor shafts have $50 \mathrm{~mm}$ diameter rollers fixed, and the yarn is wound several times using special guides onto these rollers. To enable a positive feed, each motor roller has a rubber roller pressed against it to hold the yarn. The motors have encoder wheels attached, signals from which are fed into a dual channel pulse counter. This enables the speed of the motor speeds to be monitored, as well as their ratio (percentage extension of the yarn).

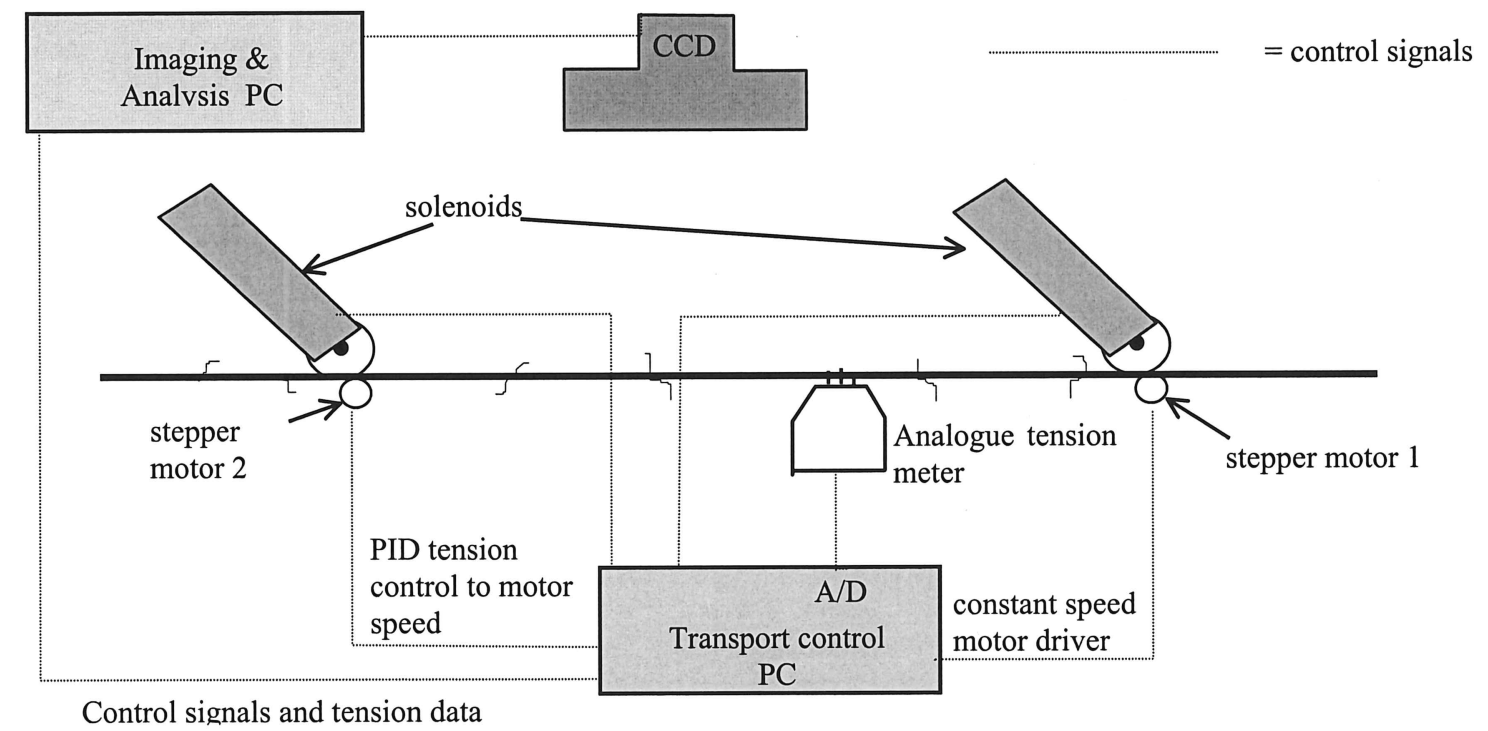

Figure 4 - Overview of the slow speed / high resolution system

Careful consideration had to be given to yarn guiding near the measuring area. Any suspended length of yarn will tend to vibrate. If the yarn section being imaged is vibrating, the effect will be that the yarn image has a periodical increase and decrease in width. This is because a lateral velocity makes the yarn appear over a wider area for a finite integration time of the CCD scan. To prevent this, two guides were placed as near to the measuring area as possible without affecting the light intensity. 


\subsection{Manual yarn inspection}

In order to verify the validity of the results from the computer system, it was necessary to use a microscope, and to manually record the nips. A sample section would be suspended across an inspection table along with a measure, and an appropriate tension was added using a weight at one end. A microscope which could slide from one end to the other was used to check the surface structure of questionable nips. Most nips could be observed with the naked eye, but some needed the yarn tension to be released to check the nip integrity.

\section{NIP MEASUREMENT METHOD}

\subsection{Requirements of the Algorithm}

The yarn diameter data has a strong component of frequency related to the nip frequency. Since the nip distances can vary considerably, this frequency content of the data is spread over a region of the discrete fourier spectrum. Any filtering of the data must not attenuate these frequencies.

Signal noise can be in the form of oscillations caused by yarn vibration, or rapid changes in the yarn diameter due to surface filaments. This region is higher in frequency than the nip frequency range, but can overlap, especially when vibration is present.

Low frequency noise is caused when the apparent diameter of the yarn changes in both the nip and open section areas together over a wider distance causing problems to any form of thresholding.

Figure 5 shows this effect, diagrammatically, and Figure 6 is a plot of the diameter of an actual intermingled yarn with superimposed orthogonal views. By inspection, it can be observed that there is no appropriate position to set a threshold, which will reliably separate all the nips in the graph from the open sections. The best position puts the threshold into the noisy region of an open section peak. Hence some form of adaptive threshold is required, or a method of removing this low frequency component.

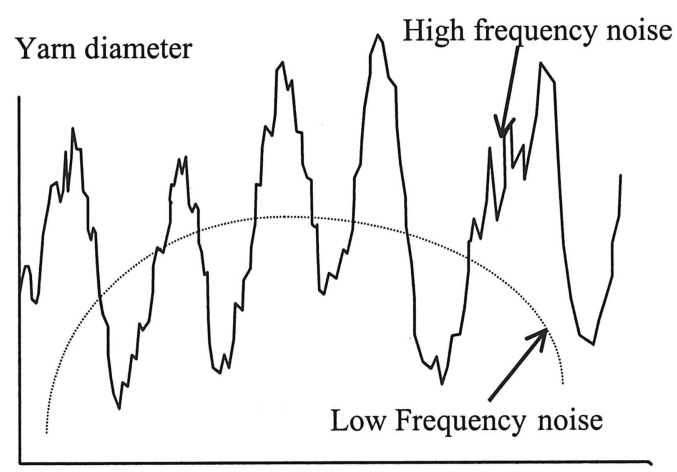

Figure 5 - The presence of noise on the data. 


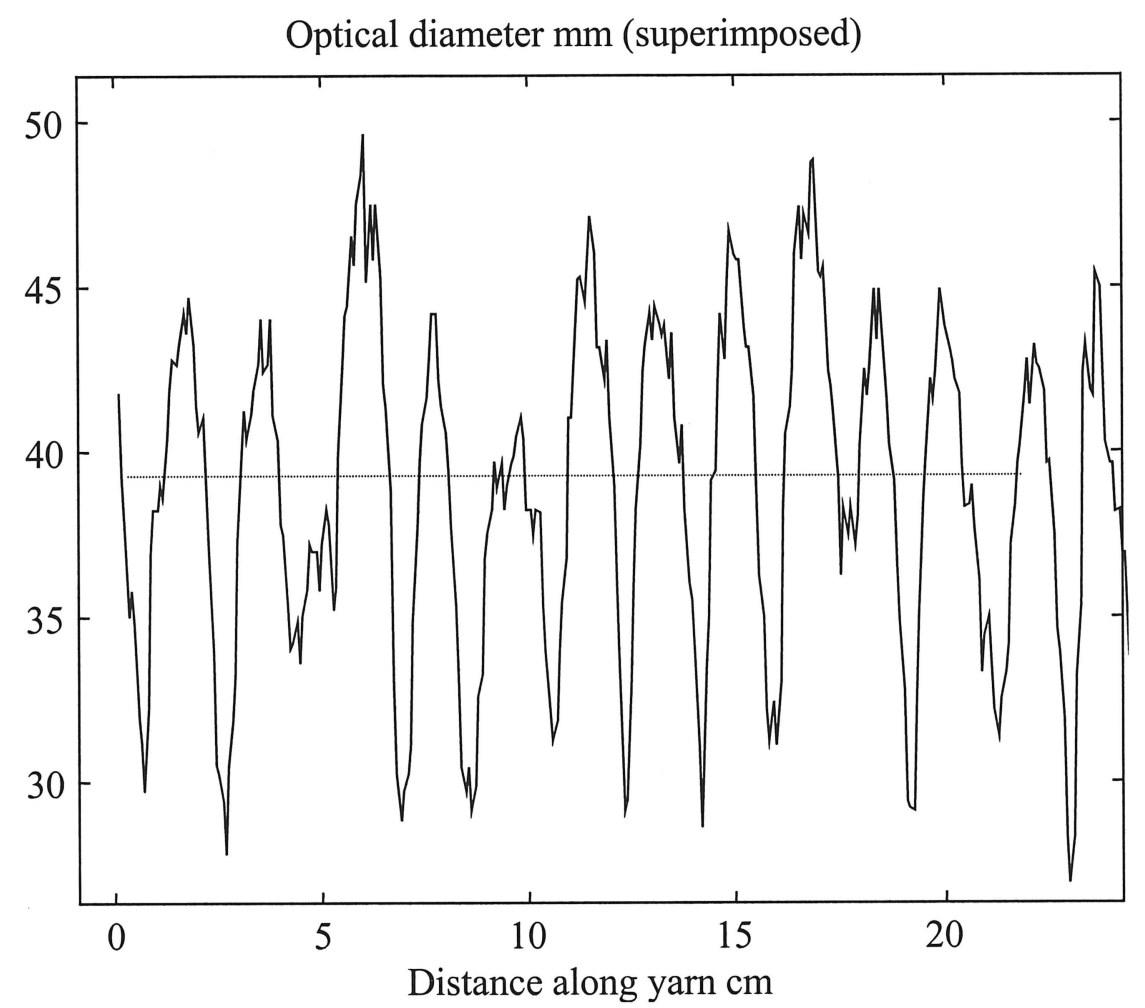

Figure 6 - Diameter profile of actual intermingled yarn illustrating the difficulty in setting a constant threshold.

The algorithm needs to avoid requiring excessive processing, and to be able to work on a continuous stream of data for possible on-line inspection.

The average nip frequency (and hence average nip pitch) can approximately be determined from the fundamental peak in the frequency spectrum of yarn diameter. However, this is only an average value and cannot provide information about individual nips. Sometimes, the mingling nozzle may fail to create a nip which needs to be identified.

The signal processing algorithm to detect nips has been developed through several stages, the first of which used a locally varying threshold technique on the yarn diameter[4]. This had drawbacks and has been superseded by the method below. Another method already developed uses the 2nd differential of the yarn profile.

\subsection{Standard Thresholding}

The first method utilised in this research was a spatial thresholding method [4]. This method applied two locally varying thresholds to the yarn diameter. If the diameter fell below the lower threshold, it was considered a nip, and if it rose above the upper threshold, it was an open section. The two thresholds were eventually replaced by just one. Figure 7 shows how the threshold is applied, and how various geometrical parameters were derived. It is worth noting that the threshold is applied on the diameter data and not the image as is implied in the figure. The difference between them is that the yarn may move sideways in the image, but its diameter isn't affected (except for high velocity movements) 


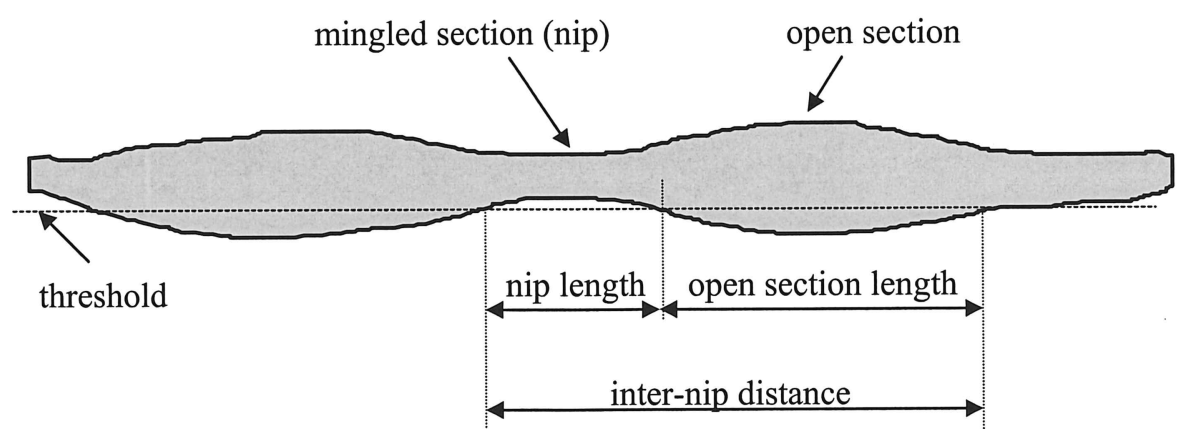

Figure 7 Mingled yarn profile

In order to overcome the low frequency noise problem the threshold was made to vary locally. In order to avoid high frequency noise, a high bandwidth low pass filter was applied to the data. Also, upon crossing the threshold the diameter would have to remain that side of the threshold for a given distance, to indicate a valid crossing. Two distance parameters were required for this; above threshold validity distance and below threshold validity distance.

The drawback of this method was that it was very sensitive to the position of the threshold and the other three parameters which need setting carefully. Thus it could not go from one set of conditions (yarn specification etc.) to another without careful adjustment. Its nip detection reliability was not satisfactory, and is included here for completeness.

\subsection{First Differential Thresholding}

The intermingled yarn can be considered as being essentially sinusoidal in diameter, with a given tolerance of frequency and amplitude along the length (Figure 8). Another approach is solving the problem of low frequency noise is to force the mean position of the sinusoid made from a nip and its adjacent sections, to a fixed level, with the sinusoid superimposed on top. This is approximately the same as removing the lower frequency components of the profile.

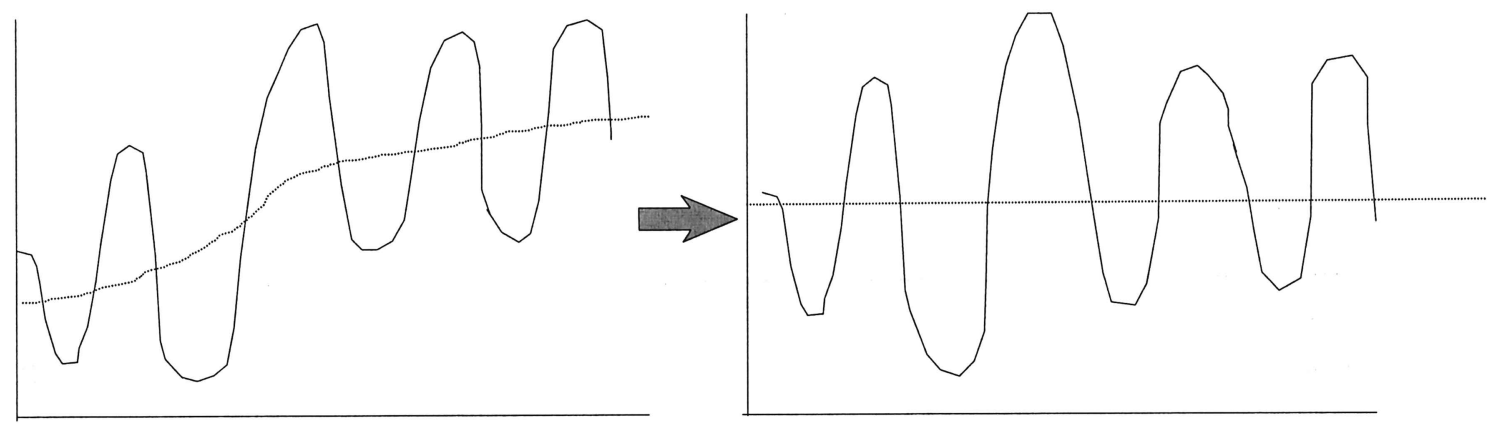

Figure 8. Forcing the mean value of the sinusoidal form of the nip profile to a constant level. 
The differentiation process removes low frequencies relative to high frequencies, and so would appear suitable in this application. The differentiating process removes the non-useful low frequency diameter variations which negatively affect the standard thresholding method, and is computationally less demanding. The first differential of a nip profile would give large values for large gradient changes in diameter. A thresholding set upon the first differential would therefore select those parts of the yarn profile which had adequate gradients in changing from nips to open sections. In order to identify the changes from open to nip sections, another threshold needs to be used, below the zero axis.

The differential method has the drawback that it amplifies high frequency 'noise' in the diameter caused by the surface filaments. This is because the amplitude of a sinusoid when differentiated is proportional to frequency :-

$$
\frac{d A \sin (w t)}{d t}=w A \cos (w t)
$$

Since the first differential is employed, there also has to be a degree of low pass filtering to avoid noise amplification. The filter bandwidth cannot be too small to cause shorter nips (composed from a higher frequency sinusoid) to be heavily attenuated, and too large so as to allow vibrational type of noise to interfere and cause false crossings of the thresholds.

From tests, it was discovered that a moving average filter (convolution with a top hat function) with a width set to a third of the average inter-nip distance gave the correct balance between low and high pass filtering.

The filter is not adaptive, and consequently nips of smaller spacing are attenuated more so than nips with larger nip to nip spacing. One disadvantage of this method is that the filtering causes some components of the yarn diameter profile to be attenuated which are not noise, and so distorting the profile to some extent.

The measure of how probable a certain thin section is on being a nip is dependent on its gradient change from open to nip section or nip to open section. Due to the complexity of the definition of this measure of nip 'probability', and the fact that absolute diameters were not being used to measure the nip appearance, the first differential method was developed into the diameter ratios method shown below.

\subsection{Second Differential Method}

The 2 nd differential of the diameter would cause the thin sections (nips) to become positive. Due to the fact that only a single threshold is needed to identify the start and finish of a nip section, this method was developed and tested against the other methods.

The second differential method has the drawback that it dramatically amplifies high frequency 'noise' in the diameter caused by the surface filaments. This is because the amplitude of a sinusoid when differentiated twice is proportional to the square of the frequency : 


$$
\frac{d^{2} A \sin (w t)}{d t^{2}}=A w^{2} \sin (w t)
$$

Therefore a substantial amount of low pass filtering is required. Tests on large lengths of yarn showed that the nip detection is sensitive to the degree of filtering, which becomes difficult to set, thus increasing the system parameter sensitivity. Also, this method preferentially identifies those nips which are shorter in length as they are composed from higher frequencies. However, the length of the nip should not be a factor provided that it is a nip.

\subsection{Diameter Ratio Method}

The ratio method uses the zero crossings of the 1st differential of the data, to determine maximum and minimum points of the yarn diameter. These correspond to open and nip sections respectively. At these points, the low-pass filtered diameter values are taken, and for any nip, its measurement is defined as the average of the diameter of the adjacent two open sections divided by the nip diameter (Figure 9). Since the first differential is employed, there has to be a degree of filtering to avoid noise amplification. The same low-pass filter for the diameter, is also applied to the differentiated signal. Experimentation found that a cut off frequency of 3 times the average nip frequency is ideal.

The advantage with this method of nip detection and measurement is that the filtering is only used to detect the presence of nips. The diameters of the unfiltered yarns can be extracted and used for nip measurement at the points where the zero crossings indicate maximas or minimas. Another benefit was that nips with only slight deviations from the open sections would cause zero crossings. They can be selectively ignored if the ratios are too large, but all nips are detected, which is useful from a yarn characterisation viewpoint.

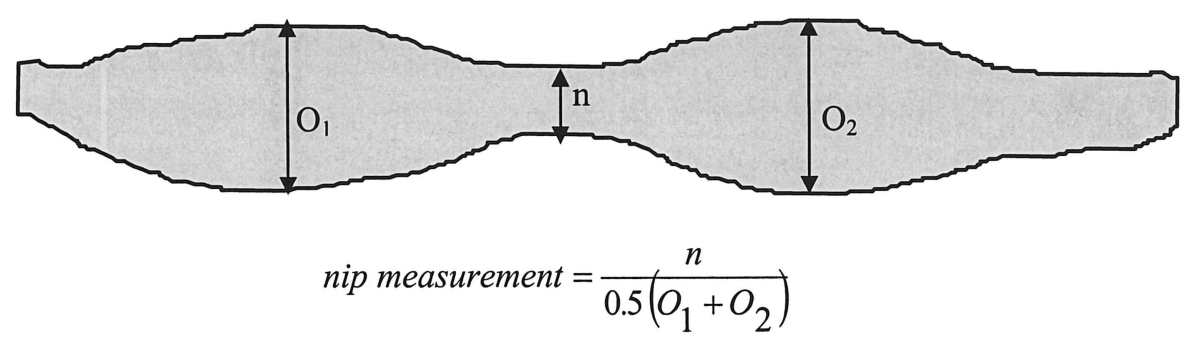

Figure 9. Definition of nip probability using the diameter ratio method.

Finally, it is desirable to provide a meaningful unit by which a nip can be measured. The 2 nd differential would effectively measure rate of change of diameter gradient, and thus provides a measure of the nips 'curviness'. The 1st differential would measure the gradients from open to nip sections. The ratio method measures the ratios of nip to open section diameters. Thus the ratio measurement is unit-less, independent of nip length, not sensitive to low frequency variations in diameter, not sensitive to noise and is not computationally demanding, can detect / reject low probable nips, does not have complex parameters to be set up, and is relatively simple. 


\section{FILTER DEVELOPMENT AND HARDWARE CONSIDERATIONS}

The ratio method requires a digital filter which differentiates the signal, and low-passes the result. The number of coefficients of the filter needs to be kept to a minimum such that a cheaper microprocessor could be capable of the task, since floating point operations are expensive in time.

It was observed that by convolving a moving average filter of ones $[1 / \mathrm{n} 1 / \mathrm{n} 1 / \mathrm{n} 1 / \mathrm{n} 1 / \mathrm{n} \ldots]$ with a differentiator $[-0.5,0.5]$, the result is a filter like this: $\left[\begin{array}{llllll}-0.5 & 0 & 0 & 0 & 0 & \ldots \\ & +0.5\end{array}\right]$. Since there are only two non-zero coefficients, it means that this filter only requires two multiplication's per digit for any required cut-off frequency. Since it is an FIR filter, its lag (group delay) is constant across the frequency response, which is also desirable.

Although implemented on a PC at present, it is feasible to implement the processing side on a dedicated DSP or micro-controller. A $16 \mathrm{MHz}$ PIC micro-controller would have 1600 machine cycles per scan to process line-scan data at $10 \mathrm{KHz}$. This is sufficient time to process the data using the ratio method.

\section{THE TESTS AND DATA ANALYSIS}

For the tests, a synthetic continuous multi-filament false-twist textured intermingled yarn was used.

\subsection{Slow speed versus manual inspection}

The first test compares the nip to nip distances observed using the microscope mount, to those obtained by the computer under slow speed scanning conditions. A $2 \mathrm{~m}$ sample was first examined using manual inspection, and the positions of its nips were recorded, along with an indication of how well formed the nips appeared (extremely poor, very poor, poor, normal). The same sample was then examined using the slow speed/high resolution computer vision system with the diameter ratio method. The distances between each nip were then compared and are shown Figure 10.

\subsection{Slow speed versus high speed long length tests}

The second test scans a yarn length of $50 \mathrm{~m}$ under slow speed scanning conditions, and compares the results with those obtained using the high speed transport at production speeds, but using the same threshold setting for the diameter method.

For the high speed test, the camera scan rate is set to $10 \mathrm{KHz}$, the yarn speed is $7.5 \mathrm{~m} / \mathrm{s}$, and the motor speed ratio (percentage slip) is 1.025 . The mean nip count per metre and standard deviation for both speeds is shown in Table 1 for both tests.

\subsection{Test results}

It is clear from Figure 10 that that for the slow speed/manual inspection test, the correlation is exact, in that if any nip was missing in one set of data, but not in the other, then the lines on the graph would become out of step by 1 nip at that point, and this isn't observed here. It was also observed that the threshold level in the diameter ratio method could selectively exclude 
those nips labelled extremely poor or very poor, which if also excluded from the manual data, would give the same exact correlation. The test proves the system for this type of yarn, and also demonstrates the use of the threshold to selectively exclude nips of poor quality.

For the second test, the mean nip count per metre and the standard deviation for both speeds are very close. The standard deviation is less than $2 \%$, showing that either the same miscalculation is happening consistently or the detection is reliable.

More importantly, the number of nips per metre is the same for tests 2 and 3 as for test 1 . This indicates that the system is working properly.

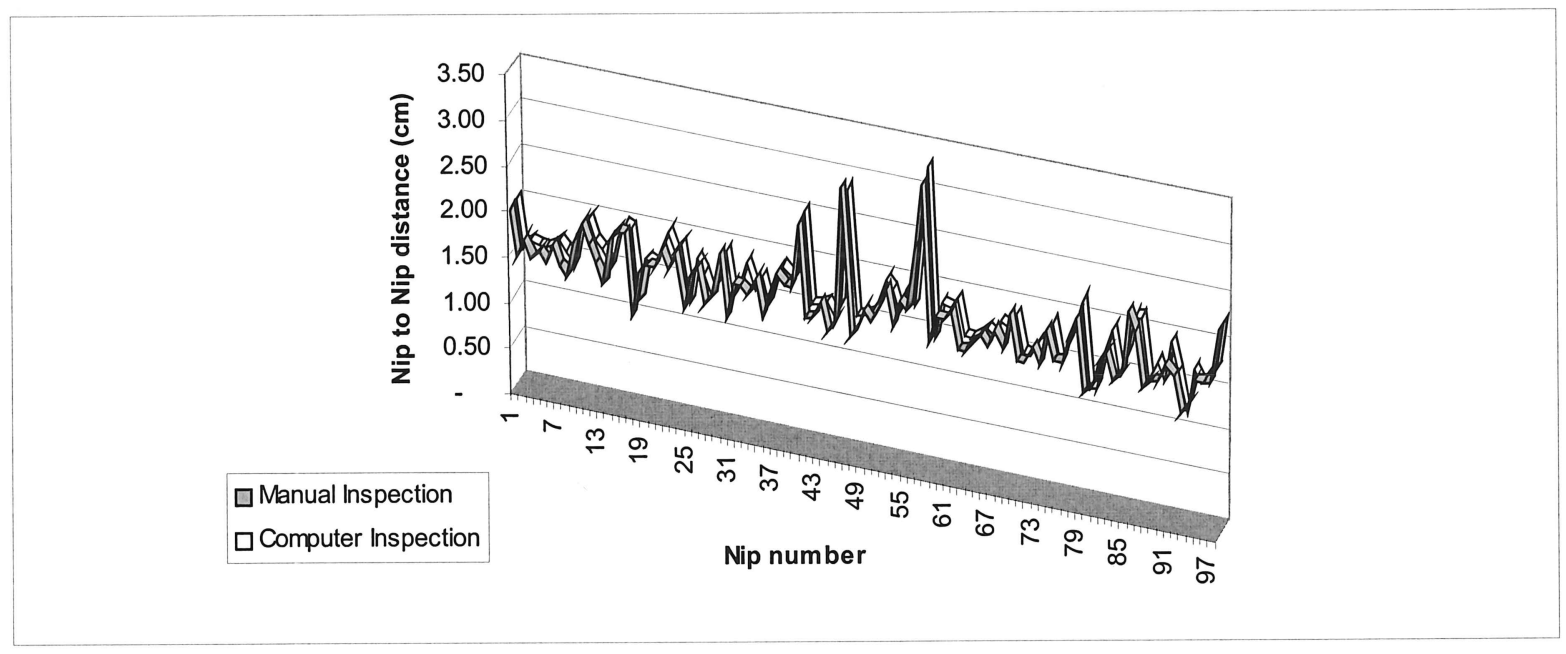

Figure 10 - Results from the low speed /manual inspection test.

Table 1 - Tests results

\begin{tabular}{lccccc}
\hline Test & $\begin{array}{c}\text { sample } \\
\text { length } \\
(\mathrm{m})\end{array}$ & $\begin{array}{l}\text { yarn } \\
\text { speed } \\
(\mathrm{cm} / \mathrm{s})\end{array}$ & $\begin{array}{l}\text { scan } \\
\text { resolution } \\
(\mathrm{mm} / \mathrm{scan})\end{array}$ & $\begin{array}{l}\text { mean } \\
/ \mathrm{m}\end{array}$ & $\begin{array}{c}\text { nips } \\
\text { Standard } \\
\text { deviation } \\
(\mathrm{nips} / \mathrm{m})\end{array}$ \\
\hline $\begin{array}{l}\text { Manual } \\
\text { inspection }\end{array}$ & 1.65 & $\mathrm{NA}$ & $\mathrm{NA}$ & 58.4 & $\mathrm{~N} / \mathrm{A}$ \\
$\begin{array}{l}\text { Low speed } \\
\text { vision }\end{array}$ & 50 & 5 & 0.172 & 56.9 & 1.58 \\
$\begin{array}{l}\text { High speed } \\
\text { vision }\end{array}$ & 50 & 750 & 0.78 & 54.2 & 1.64 \\
\hline
\end{tabular}

\section{CONCLUSIONS}

The tests show that nip detection using machine vision is reliable provided that an algorithm is used which can overcome low frequency and high frequency. An algorithm has been 
developed (the ratio method), which gives a measure of the probability that a thin section is a nip. It is able to detect those nips that are only partially formed and segregate them if required from the well formed nips. The algorithm is also not computationally demanding, and relies on quickly computable filters to differentiate the yarn profile. The measure of nip probability is unit-less, simple to understand, and impartial to the length of the nips. The algorithm is capable of both high and low yarn speed conditions. The system is shown to be technically capable of in-process inspection, since the speed used in the tests $(7 \mathrm{~m} / \mathrm{s})$ is of the order of those used in production

\section{REFERENCES}

1. Wray G. R. and Acar M. Supersonic Jet Texturing of Yarns, Proceedings of the IMechE, Part B: Journal of Engineering Manufacture, 1990, Vol. 204, 71-89.

2. Demir A. and Acar M. An Insight into the Intermingling Process, in: Textiles: Fashioning the Future, Manchester, 1989, 169-191.

3. Wegner E and Hedwig R, Forschungsberichte des Landes Nordrhein-Westfalen, No.2007,1968

4. Acar M, Jackson MR and Millman MP, High speed yarn characterisation and monitoring using a vision based scanning system, Conf on Textile Process Control, 2001, Manchester University UMIST, 1995.

5. Millman M.P, Acar M and Jackson M.R., Optical Resolution Limits and Digital Image Processing Techniques for Yarn Image Acquisition., Mechatronics'96 - International Conference, 1996, University of Minho, Vol. 2, 2.115 -2.122 